\title{
Einschlafen an der Brust - schlechte Gewohnheit oder sinnvolle Einrichtung der Natur?
}

\author{
Sibylle Lüpold, Krankenschwester und Stillberaterin, Herrenschwanden
}

\section{Wie schläft ein Baby am besten ein? Wo soll es (ein)schlafen? Wann soll es lernen, alleine und/oder ohne Hilfsmittel einzuschlafen? Darf es an der Brust einschlafen? Da die Empfehlungen diesbezüglich sehr widersprüchlich sind, ist es hilfreich, Eltern zu ermutigen, bei Verunsicherung nach der fachlichen Begründung zu fragen.}

\section{Ein Fallbeispiel}

„Meine Tochter ist 6 Monate alt und bisher immer an der Brust eingeschlafen. Gestern war ich zur Kontrolle bei der Mütterberaterin und berichtete ihr davon. Sie riet mir, mein Kind ab sofort nicht mehr an der Brust einschlafen zu lassen; das sei eine schlechte Gewohnheit, die ich später nur noch schwer abgewöhnen könne. Also stillte ich meine Tochter gestern Abend vor dem Zu-BettGehen und legte sie dann noch wach in ihr Bettchen. Sie regte sich dabei aber furchtbar auf und schrie so lange, bis ich sie herausnahm und herumtrug. Als sie sich wieder beruhigt hatte, stillte ich sie noch einmal und dabei schlief sie friedlich ein. Habe ich es bisher falsch gemacht und wie wird meine Tochter lernen, ohne Brust einzuschlafen?“

\section{Welche Vorteile bietet das Einschlafen an der Brust?}

Nach der Geburt ist es für den Beginn einer erfolgreichen Stillbeziehung sehr förderlich, wenn das Neugeborene seiner Mutter möglichst rasch im Haut-zuHaut-Kontakt auf den Bauch und innerhalb kurzer Zeit zum ersten Mal an die Brust gelegt wird. Aus verschiedenen Gründen ist es in den ersten Tagen (und auch später) wichtig, ein Kind häufig und nach Bedarf zu stillen: So kann es das Saugen an der Brust optimal erlernen, die mütterliche Milchbildung wird gut angeregt und eine unangenehme Brustdrüsenschwellung wird verhindert [1].

Da ein Neugeborenes in den ersten Wochen tagsüber noch viel schläft, ist es bei einem Stillkind ganz normal, dass es auch regelmäßig an der Brust einschläft.

Das Saugen ist einerseits ermüdend, andererseits wirken der Körperkontakt (aufgrund der ausgeschütteten Endorphine und Oxytozin) und Inhaltsstoffe in der Muttermilch beruhigend bis einschläfernd. Aber auch die Mutter kann sich beim Stillen - sofern dieses gut läuft und schmerzfrei ist - entspannen. Idealerweise lernen stillende Mütter nach der Geburt, bequem liegend zu stillen, damit sie sich dabei optimal erholen können.

Mit der Zeit verschieben sich die Schlafphasen des Säuglings zunehmend in die Nachtstunden [2]. Interessant ist hierbei, dass die Zusammensetzung der Muttermilch sich nicht nur mit dem Alter des Kindes verändert, sondern auch innerhalb eines Tages. Am Morgen finden sich Stoffe, die wach machen, während abends schlafmittelähnliche Substanzen darin enthalten sind [3]. Dazu gehören verschiedene Aminosäuren, u.a. Tryptophan, das im kindlichen Organismus zu Melatonin umgebaut wird. Melatonin hat eine schlaffördernde Wirkung.
Muttermilch auch als „Schlafmittel“ zu nutzen, ist aus biologischer Sicht deshalb äußerst sinnvoll.

Die meisten Mütter berichten davon, dass ihr Kind an der Brust am besten einschläft, während es beim Tragen viel länger dauert. Alleine ins Bettchen gelegt zu werden und ohne Körperkontakt zur vertrauten Bezugsperson einzuschlafen, können nur wenige Säuglinge vor dem ersten Geburtstag. Die meisten reagieren, auch wenn sie eigentlich müde sind, mit heftigem Protest und beruhigen sich erst im Arm der Mutter oder des Vaters wieder.

Evolutionsbiologen gehen davon aus, dass Menschenkinder über den Großteil der Menschheitsgeschichte sehr häufig und auch sehr lange (2-7 Jahre) gestillt wurden und es ist anzunehmen, dass sie auch an der Brust einschliefen $[4,5,6]$. In einer ursprünglichen Jäger- und Sammlergemeinschaft, die im Freien übernachtete, war es notwendig, einen Säugling nachts zu stillen und dadurch rasch zu beruhigen, damit er nicht schrie und wilde Tiere anlockte. Diese Art der nächtlichen Kinderbetreuung wird erst seit einigen Jahrzehnten in modernen westlichen Gesellschaften hinterfragt.

Das Einschlafen an der Brust und auch das nächtliche Stillen bieten wesentliche Vorteile für Mutter und Kind:

- Die meisten Stillkinder schlafen an der Brust gut ein. Auch die Mutter 
wird müde und findet besser in den

Schlaf (was natürlich von Nachteil ist, wenn sie danach noch fit sein möchte).

- Nachts ist der mütterliche Prolaktinspiegel erhöht und durch das nächtliche Stillen verbessert sich die Milchproduktion. Daran ist auch der zusätzliche Körperkontakt beteiligt.

- Außerdem gedeihen Kinder, die rund um die Uhr gestillt werden, besser. Die Wachstumshormone werden größtenteils nachts ausgeschüttet [7].

- Umgekehrt kann sich durch ein plötzliches und frühes Durchschlafen des Kindes die Milchproduktion der Mutter reduzieren und die Gewichtskurve des Kindes abflachen [8]. Auch ein schmerzhafter Milchstau kann dadurch begünstigt werden [1].

- Stillkinder, die abends und nachts gestillt werden, genießen eine deutlich längere Gesamtstillzeit. Frühes Durchschlafen begünstigt hingegen ein früheres Abstillen [9].

- Gestillte Kinder befinden sich häufiger im aktiven REM-Schlaf [2, 7], der für ihre Entwicklung wichtig ist. Im REM-Schlaf sind sowohl Atmung als auch Gehirnaktivität erhöht. Risikokinder für plötzlichen Kindstod (SIDS) sind gerade in den Tiefschlafphasen gefährdet, dass die Atmung ausfällt. Das Stillen stellt auch aus anderen Gründen einen Schutzfaktor für SIDS dar [1].

- Schläft ein gestilltes Kind neben der Mutter im Bett (Sicherheitsvorkehrungen beachten!) oder im Bettchen daneben, kann es auf einfache Weise wieder in den Schlaf gestillt werden, sollte es erwachen.

- Da sich die Schlafphasen gemeinsam schlafender Mutter-Kind-Paare angleichen, weckt das Kind seine Mutter nicht aus dem Tiefschlaf. Stillende Mütter machen die Erfahrung, dass sie jeweils wach werden, kurz bevor ihr Kind sich bewegt und nach der Brust sucht [7].

- Durch das unmittelbare nächtliche Stillen werden folglich auch die nächtlichen Schreiphasen reduziert, wenn nicht ganz verhindert. Manche
Mütter erinnern sich am Morgen nicht daran, ob und wie oft sie nachts gestillt haben.

- Die sichere Bindung zwischen Mutter und Kind und das gegenseitige Vertrauen werden durch die zusätzliche Zuwendung unterstützt.

- Stillen ist gerade auch nachts eine optimale Möglichkeit, körperliches

Unwohlsein rasch zu beseitigen und emotionale Bedürfnisse zu erfüllen.

- Umgekehrt sind Hunger und Schreienlassen für den Säugling höchst beängstigende Zustände [12].

- Mütter, die in der Lage sind, ihr Baby auf einfache Weise in den Schlaf zu begleiten, fühlen sich in ihrer Rolle bestärkt. Das kann auch durch die Fütterung mit der Flasche und eine feinfühlige Betreuung erfolgen.

- Die empfängnisverhütende Wirkung durch das Stillen (Laktationsamenorrhoe) ist nur gewährleistet, wenn u.a. auch nachts regelmäßig gestillt wird [1].

Manche Fachleute sind der Ansicht, dass ein Kind nach sechs Monaten nicht mehr auf nächtliche Nahrung angewiesen ist. Ganz anders sieht das der Anthropologieprofessor und Leiter eines Forschungszentrums zum Schlafverhalten von Mutter und Kind, Dr. James McKenna: Stillkinder wachen nachts häufiger auf als Babys, die mit künstlicher Säuglingsmilch ernährt werden.

\section{Muttermilch hat im Gegensatz zu}

Kuhmilch die ideale Zusammensetzung für das ständig wachsende Gehirn des Säuglings, dessen Größe sich im ersten Lebensjahr mehr als verdoppelt [13].

Muttermilch ist im Vergleich zu anderen Säugetieren kohlehydratreicher, aber protein- und fettärmer. Sie verlässt den Magen bereits nach ca. 20 Minuten und wird innerhalb von 1-2 Stunden verdaut [14]. Da sich ein Baby noch viel häufiger im REM-Schlaf befindet als ein Erwachsener und da im REM-Schlaf die Gehirnaktivität auf Hochtouren läuft, kann ein Kind auch nach 6 Monaten noch auf nächtliche „Imbisse“ angewiesen sein.
Stillen ist aber nicht nur Ernährung mit Muttermilch.

Stillen löscht den Durst, senkt den Blutdruck, optimiert die Atmung, verbessert das Wohlbefinden, hilft bei Erkrankungen, lindert Schmerzen, spendet Trost usf. So ist es gerade beim älteren Kind nicht nur der Hunger, der das Bedürfnis auslöst, an der Brust einzuschlafen oder der nachts zum Aufwachen führt. Gerade die mit dem Durchbrechen der ersten Zähne verbundenen Schmerzen, emotionale Faktoren und die zunehmende Bindung führen zu nächtlichen Schlafunterbrechungen. Das Saugen an der Mutterbrust und die damit verbundene intensive Nähe helfen dem Kind, sich wieder zu beruhigen und ein- oder weiterzuschlafen.

\section{Schlafentwicklung}

Die weitverbreitete Annahme, dass ein Baby spätestens mit 6 Monaten durchschlafe, entspricht nicht der Realität.

Die Schlafentwicklung verläuft nämlich keineswegs linear. Sehr viele Eltern (auch solche, deren Kind schon abgestillt ist), die sich drei Monate nach der Geburt darüber freuen, dass das Kind nachts schon mehrere Stunden am Stück schläft, stellen im 2. Lebenshalbjahr verunsichert fest, dass es nun wieder häufiger wach wird. Dieses Phänomen ist aber kein Hinweis auf eine „falsche“ Erziehung, sondern ist als durchaus positiver Entwicklungssprung zu werten. Brazelton spricht von sogenannten „Touchpoints“, d.h. kritischen Meilensteinen in der kindlichen Entwicklung und damit verbundenen, möglichen Störungen des Familiensystems. Dazu gehört auch das vermehrte nächtliche Aufwachen zwischen 8 und 12 Monaten [15]. Wenn Eltern darauf vorbereitet werden und die Veränderung richtig interpretieren, können sie besser damit umgehen. 


\section{Gewöhnung an das Einschlafstillen?}

In Deutschland werden Kinder durchschnittlich 6,9 Monate lang gestillt [16]. Das heißt, ein Großteil der Säuglinge wird bereits nach wenigen Monaten definitiv abgestillt, wodurch sich die Frage erübrigt, wie sie davon entwöhnt werden sollen, an der Brust einzuschlafen.

Wird ein Kind länger als ein halbes Jahr gestillt, kann es vorkommen, dass es schreit, wenn die Mutter das gewohnte Einschlafritual verändern möchte. Der Mutter nun vorzuwerfen, sie habe den Zeitpunkt verpasst, ihrem Kind diese „lästige Gewohnheit“ abzutrainieren und es werde nur noch schwer lernen, anders einzuschlafen, ist sehr kurzsichtig.

\section{Die Entwicklung des Kindes bleibt im Idealfall ja keineswegs stehen. Ein sicher gebundenes Kind strebt von sich aus die Autonomie an, wenn es dazu bereit ist.}

Von einem Baby oder Kleinkind zu erwarten, dass es alleine einschlafen möchte, wenn es bisher auf die Nähe der Mutter oder des Vaters und das beruhigende Saugen an der Brust vertrauen durfte, ist jedoch oft zu viel verlangt. Warum sollte es von sich aus eine so angenehme und geborgenheitsvermittelnde Einschlafmethode aufgeben - und das in einem Alter, in dem entwicklungsbedingte Ängste (die Fremdenangst, später die Trennungsangst) vorherrschend sind?

Da die wenigsten Fachleute und auch Eltern Kinder kennen, die länger als zwei oder drei Jahre gestillt wurden, fehlt die Erfahrung, wie die kindliche Schlafentwicklung verläuft, wenn nicht aktiv durch irgendwelche Erziehungsmethoden eingegriffen wurde. In der Stillberatung der La Leche League finden sich vermehrt solche Beispiele. Mütter von gestillten Kindern, die so lange an der Brust einschlafen durften, wie sie dies nötig hatten, berichten davon, dass das
Einschlafritual mit zunehmendem Alter und fortschreitender Entwicklung des Kindes ohne Tränen verändert werden konnte.

\section{Die meisten Stillkinder stillen sich} zwischen dem 2. und 4. Geburtstag von selbst ab, womit auch das abendliche und nächtliche Stillen wegfällt.

Wenn Mütter nicht so lange Geduld haben, dürfen sie selbstverständlich auch aktiv eine Veränderung ansteuern. Mit zunehmender Sprachentwicklung können einem Kind abends zum Einschlafen attraktive Alternativen zur Brust geboten werden: Zusammen ein Bilderbuch schauen und eine Geschichte erzählen oder gemeinsames Singen sind bewährte Methoden. Wenn das Kind weiterhin in Gegenwart einer nahen Bezugsperson einschlafen darf, wird es den Verzicht auf die Brust besser akzeptieren können.

Manche Mütter berichten jedoch, dass das Einschlafstillen jeweils sehr lange dauert und sie dabei ungeduldig werden. Gerade bei Wachstumsschüben kann es vorkommen, dass Babys sehr lange an der Brust saugen möchten, um die Milchproduktion der Mutter anzukurbeln. Sobald die Milch besser fließt, verkürzen sich die Stillmahlzeiten wieder.

Es kann aber auch sein, dass ein Kind sehr lange gestillt werden möchte und dabei nur schwer in den Schlaf findet, weil es große äußere oder innere Veränderungen durchmacht oder weil es die Trennung von seiner Mutter verhindern möchte.

Einschlafen ist eine Trennungssituation und solange das Kind an der Mutterbrust saugt, fühlt es sich sicher.

Wenn ein Kind hingegen mehrmals die Erfahrung gemacht hat, dass es nachts alleine aufgewacht ist, kann ihm das Vertrauen fehlen, dass seine Mutter auch wirklich in seiner Nähe bleibt. Kinder, die von Geburt an bei den Eltern schlafen und verinnerlicht haben, dass sie auch nachts nicht alleine sind, verknüpfen das Einschlafen und die Dunkelheit nicht mit Einsamkeit und damit verbundenen Ängsten. Solche Kinder schlafen oft entspannter ein.

Zurück zur Frage, ob das Einschlafen an der Brust nicht doch eine schlechte Gewohnheit ist, die besser schon von Anfang an vermieden werden sollte: Die Tatsache, dass ein Baby an der Brust gut und schnell einschläft, ist nicht eigentlich der störende Faktor. Kritisiert wird diese Methode deshalb, weil ein Kind, das abends an der Brust einschläft, auch nachts, wenn es erwacht, danach verlangt. Es habe sich daran gewöhnt, nur stillend einzuschlafen. Umgekehrt rufe ein Kind, das früh schon lernt, alleine einzuschlafen, auch beim nächtlichen Aufwachen nicht nach den Eltern, sondern finde alleine wieder in den Schlaf.

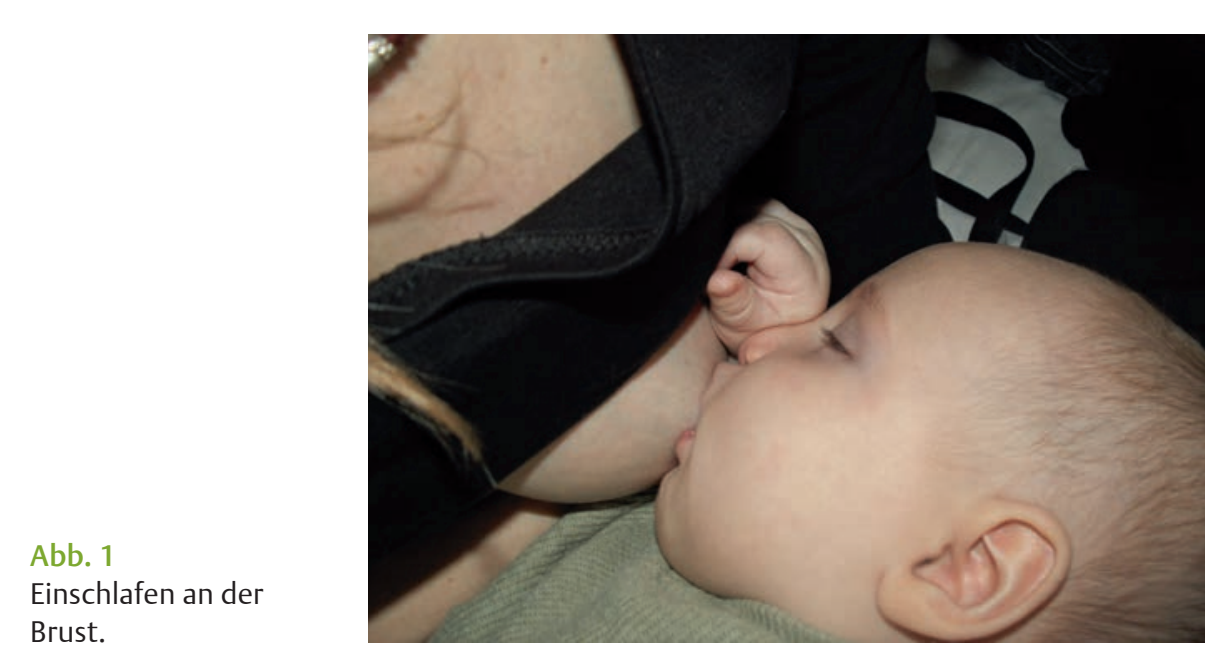


Man erhofft sich also, durch das Abgewöhnen des Einschlafstillens ein rasches Durchschlafen zu erzielen.

In der Tat berichten manche Mütter, die beschlossen haben, ihr Kind nicht länger in den Schlaf zu stillen, davon, dass es von da an auch durchgeschlafen habe. Viele Kinder wachen aber im Verlauf ihrer Entwicklung aus unterschiedlichen Gründen nachts trotzdem wieder auf und rufen nach den Eltern. Das heißt, durch das nächtliche Abstillen kann nicht verhindert werden, dass ein sicher gebundenes Kind nachts wach wird und seine Bedürfnisse anmeldet.

Längerfristig betrachtet lernen auch Kinder, die an der Brust einschlafen, früher oder später alleine ein- und durchzuschlafen. Die vermehrte nächtliche Zuwendung und der zusätzliche Körperkontakt wirken sich zweifellos positiv auf die kindliche Entwicklung aus.

\section{Fazit}

- Die meisten Stillkinder schlafen an der Brust sehr gut ein. Fachleute, die davon abraten, obwohl die Mutter mit der Situation zufrieden ist, verunsichern die Mütter nur unnötig und raten zu Maßnahmen, die sich sowohl auf die Stillbeziehung als auch auf die Mutter-Kind-Bindung störend auswirken können.

- Einschlaf- und nächtliches Stillen bieten für Mutter und Kind zahlreiche Vorteile. Der einzige Grund, etwas daran zu ändern, ist die berechtigte Tatsache, dass die Mutter sich dabei nicht wohl fühlt.

- Natürlich kann ein Kind daran gewöhnt werden, ohne Brust einzuschlafen; das dient jedoch ausschließlich den Eltern und sollte nicht im angeblichen Interesse der kindlichen Entwicklung empfohlen werden.

\section{Literatur}

1 Mohrbacher N., Stock J: Handbuch für die Stillberatung, La Leche League Deutschland, 2002

2 Largo R: Babyjahre, Piper München, 2007

3 Sanchez CL et al.: Nutritional Neuroscience 2009; 12 (19), 2-8

4 Renz-Polster H: Kinder verstehen, Kösel München, 2010

5 Dettwyler K: A Time to Wean, www.ueberstillen.org

6 Blaffer Hrdy S: Mutter Natur, Berlin Verlag 2010

7 Sears W: Schlafen und Wachen, La Leche League International 2005

8 Ball HL: Breastfeeding, bed-sharing and infant sleep. Birth; 30 (3): 181-188

9 McKenna J, Mosko S et al.: Bedsharing promotes breastfeeding. Pediatrics; 100 : 214-219

10 McKenna J: Sleeping with your Baby, Platypus Media, Washington, 2007

11 Lüpold S: Ich will bei euch schlafen! Urania Stuttgart, 2009

12 Brisch KH: Safe - Sichere Ausbildung für Eltern, Klett-Cotta 2012

13 McKenna J: Jedes Kind lernt irgendwann zu schlafen, WirbelWind 2/2008

14 Dettwyler K: How often do other species nurse? www.ueberstillen.org

15 Brazelton TB, in: Brisch KH, Hellbrügge Th (Hrsg): Der Säugling - Bindung, Neurobiologie und Gene, Klett-Cotta 2008

16 Deutscher Hebammenverband (Hrsg): Praxisbuch: Besondere Stillsituationen, Hippokrates 2012

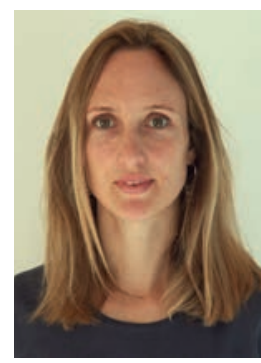

Sibylle Lüpold

Stillberaterin Aareweg 18e 3037 Herrenschwanden bei Bern (Schweiz) sibylle.luepold@gmail.com 